\title{
The Standard-Model Extension and Gravitational Tests
}

\author{
Jay D. Tasson
}

Department of Physics and Astronomy, Carleton College, One North College St., Northfield, MN 55057, USA; jtasson@carleton.edu

Academic Editor: Eberhard Widmann

Received: 1 September 2016; Accepted: 8 October 2016; Published: 27 October 2016

\begin{abstract}
The Standard-Model Extension (SME) provides a comprehensive effective field-theory framework for the study of CPT and Lorentz symmetry. This work reviews the structure and philosophy of the SME and provides some intuitive examples of symmetry violation. The results of recent gravitational tests performed within the SME are summarized including analysis of results from the Laser Interferometer Gravitational-Wave Observatory (LIGO), sensitivities achieved in short-range gravity experiments, constraints from cosmic-ray data, and results achieved by studying planetary ephemerids. Some proposals and ongoing efforts will also be considered including gravimeter tests, tests of the Weak Equivalence Principle, and antimatter experiments. Our review of the above topics is augmented by several original extensions of the relevant work. We present new examples of symmetry violation in the SME and use the cosmic-ray analysis to place first-ever constraints on 81 additional operators.
\end{abstract}

Keywords: gravity; Lorentz symmetry; CPT symmetry

\section{Introduction}

Our present description of nature is based on two enormously successful theories: General Relativity (GR), a classical theory describing all gravitational phenomena, and the Standard Model (SM) of particle physics, which provides a quantum description of all other interactions. It is widely expected that these theories are merely the low-energy limit of some more fundamental theories that would take over as the characteristic energies involved in experiments approach the Planck scale, $10^{19} \mathrm{GeV}$. Experimental information to guide the development of a Planck-scale theory would, by conventional thinking, come from Planck-energy experiments, which are likely to remain infeasible far into the future. An alternative approach is to search for small deviations from known physics (the SM and GR) in present-day experiments, with the hope that small deviations, if found, would encode information about the underlying theory.

Lorentz symmetry, the idea that physical results are unchanged under rotations and boosts of the system, and CPT symmetry, the associated invariance of the system under the combination of discrete symmetries of charge conjugation, parity, and time reversal, are pillars of both the SM and GR. Hence, violations of these symmetries, if found, would provide a novel signal of new physics. Moreover, the possibility of violations of these symmetries has been demonstrated in candidates for the underlying theory, like strings $[1,2]$.

The systematic search for Lorentz and CPT violation using the comprehensive effective field theory based framework of the Standard-Model Extension (SME) [3-8] provides a method of searching for Planck-suppressed effects in known physics in a complete and organized way. In a nut shell, the SME adds to known physics all Lorentz and CPT violating effects at the level of the action. The terms added to the action of the SM and GR to form the SME are generated from Lorentz and CPT violating operators acting on SM and GR fields along with coefficients for Lorentz and CPT violation that parameterize the amount of symmetry violation in the theory. The addition of Lorentz and CPT violating terms 
can be thought of as a series expansion about known physics in ever increasing mass dimension of the operators involved. The SME coefficients can then be sought in experiments. Over 1000 limits on SME coefficients have been set via experiments and observations [9], but much remains to be explored, particularly in the case of the so-called non-minimal operators of mass dimension greater than four, where few constraints have been set by the direct analysis of experimental data. It should be emphasized that the SME is a test framework designed for a broad search for yet-unobserved symmetry violation, a philosophy that is quite different from model building. Though the SME is unique in providing a comprehensive test framework at the level of the action [10], other approaches to the study of Lorentz and CPT violation exist [11] and the idea of a general test framework over specific models has philosophical resonance with efforts to parameterize deviations from GR [12,13].

In the next section, Section 2, a basic introduction to both Lorentz and CPT symmetries is provided along with introductory-physics level examples that illustrate behaviors that arise from violations of these symmetries. Following these basics, Section 3 provides a summary of the SME philosophy and the effective-field theory based structure it employs. An alternative and somewhat more in-depth treatment paralleling Sections 1-3 can be found in Ref. [10] along with some more general review of other areas of activity within the SME. Section 4 reviews recent experimental results and phenomenological proposals that are connected with gravitational physics. One recent work [14] places tight initial constraints on 74 Lorentz-violating operators of mass dimension 6 and 8 in the pure-gravity sector. In Section 5, we extend this analysis to obtain another 81 tight initial constraints on dimension 10 operators. Throughout this work, we use natural units and metric signature +2 .

\section{Symmetry Violation}

In this section, we consider several examples of symmetries and their violation. Though the examples are comparatively simple, the nature of these examples will map directly onto the SME structure in Section 3. Lorentz symmetry contains both rotations and boosts. In the first subsection, we will appeal to the visual nature of rotation invariance and consider examples of rotation invariance and rotation-invariance violation as examples of the Lorentz-symmetry case. In the second subsection, $\mathrm{CPT}$ symmetry and CPT violation will be considered.

\subsection{Rotations}

To begin our example, consider the classical, nonrelativistic Lagrangian for a particle of mass $m$, position $\vec{r}$, and charge $q$ in a magnetic field $\vec{B}$ given by a vector potential $\vec{A}(\vec{B}=\vec{\nabla} \times \vec{A})$ :

$$
L=\frac{1}{2} m \dot{\vec{r}}^{2}+q \dot{\vec{r}} \cdot \vec{A}
$$

We will first do what is known as an observer transformation on this system, in this case a rotation. This corresponds to the experimenter turning his or her head and taking their coordinates with them. This transformation is carried out by acting with the standard rotation matrix $\mathbf{R}$ on all vector components such that the components of a generic vector $\vec{V}$ transforms as $V_{j} \rightarrow \vec{V}_{j^{\prime}}=R_{j^{\prime} j} V_{j}$. Doing this transformation to all vector components in (1) reveals that it is form invariant. This is a signal that the theory in (1) possesses "observer-rotation invariance" as might have been expected. In other words, the outcome of experiments governed by (1) does not depend on the coordinates used.

To see even more explicitly that the theory is invariant, we can perform the following series of steps: (i) Set up a system with some initial conditions; (ii) Calculate the final configuration using the original theory, where in the example above, the acceleration of the particle $\vec{a}$ suffices as a proxy for the final configuration; (iii) Apply the symmetry transformation to the result; (iv) Apply the symmetry transformation to the initial conditions; and (v) Calculate the final configuration using the transformed theory. As shown in Figure 1, when these steps are applied for observer rotations, the results of steps (iii) and (v) match, reflecting the obvious observer-rotation invariance of the system. 


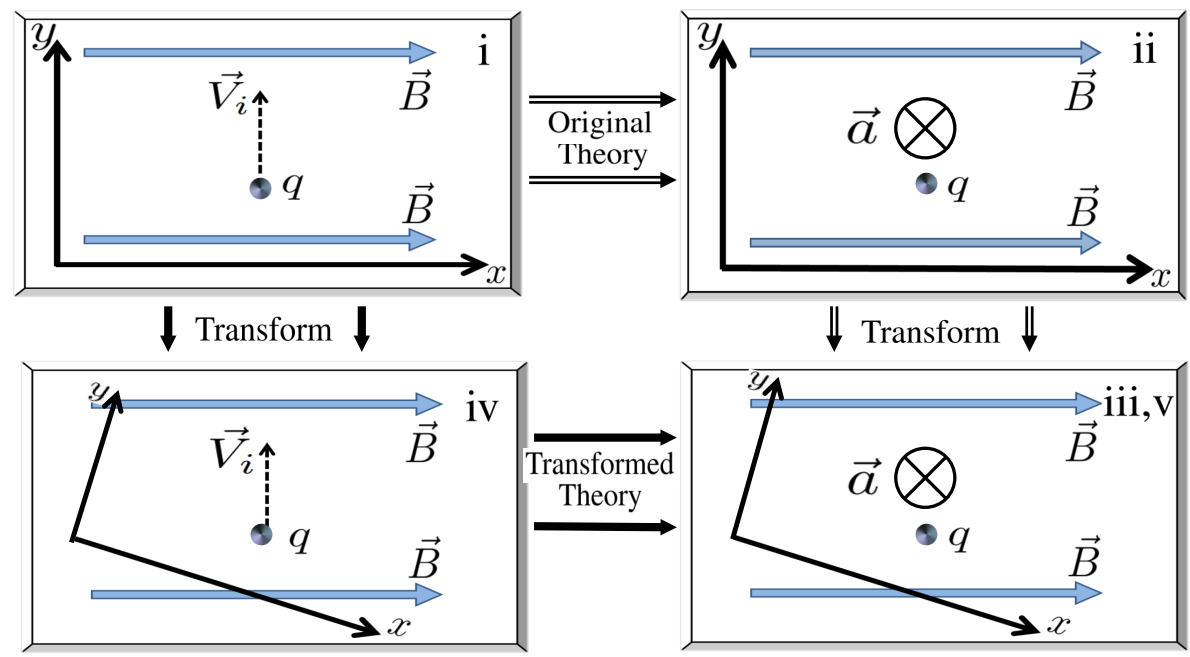

Figure 1. Illustration of observer symmetry in a sample system.

We can next apply the same procedure for a particle rotation. This procedure leaves the observer and the coordinates fixed but rotates all fields. Operationally, the procedure is carried out the same way on this rotation-invariant system, and as result of the symmetry, the outcome will be identical. While we could draw this procedure in an identical way, observer and particle transformations will be distinct when spacetime symmetries are broken, and we draw the pictures in Figure 2 for the particle transformation case somewhat differently to highlight the difference between rotating the coordinates and rotating the physical system.

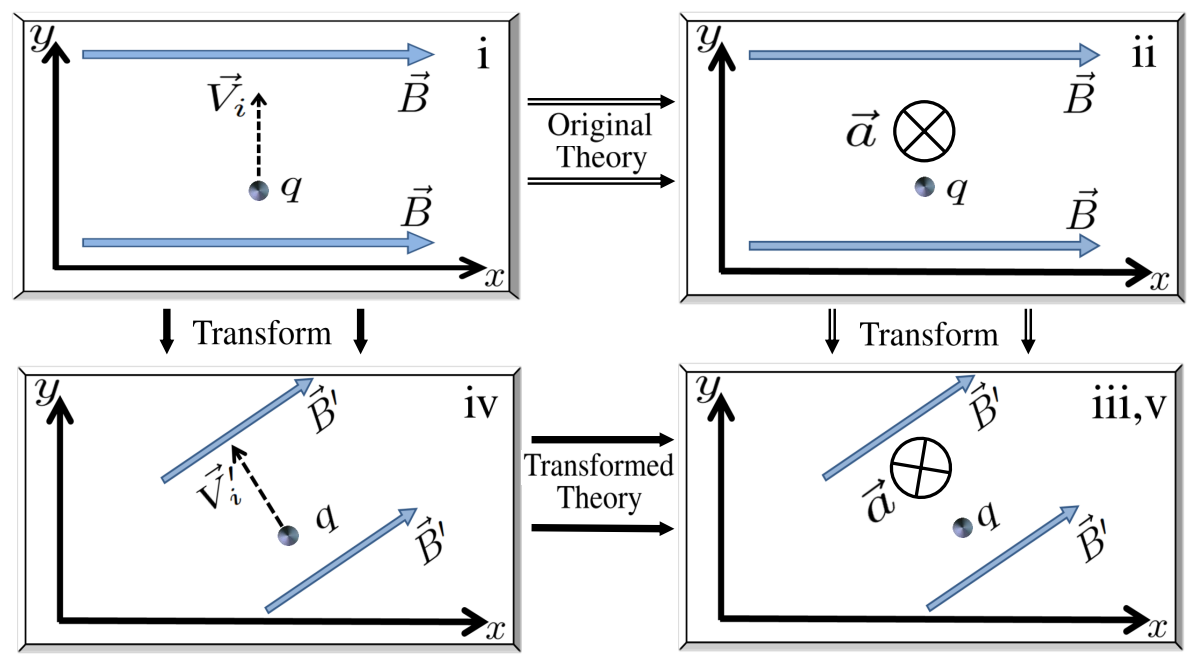

Figure 2. Illustration of particle-rotation symmetry in a sample system.

We can now use this system as a toy model for broken Lorentz invariance. Suppose that the experimenter were unaware of the physics generating the vector potential and hence the magnetic field. Perhaps it exists on a much larger scale than their lab. If they now perform a particle rotation on "the system," they will rotate the apparatus in their lab, but not the magnetic field. Here, the transformed theory will be

$$
\begin{aligned}
L & =\frac{1}{2} m \dot{\vec{r}}^{2}+q \dot{\vec{r}}^{\prime} \cdot \vec{A} \\
& =\frac{1}{2} m \dot{\vec{r}}^{2}+q \dot{\vec{r}} \mathbf{R}^{T} \cdot \vec{A}
\end{aligned}
$$


where $\mathbf{R}^{T}$ is the transposed rotation matrix. Note that the theory is no longer particle-rotation invariant as the form has changed. Applying this transformation to our cartoon example leads to the situation shown in Figure 3 in which the acceleration found in the transformed system is different (acceleration is smaller under the conditions shown) than the original system.

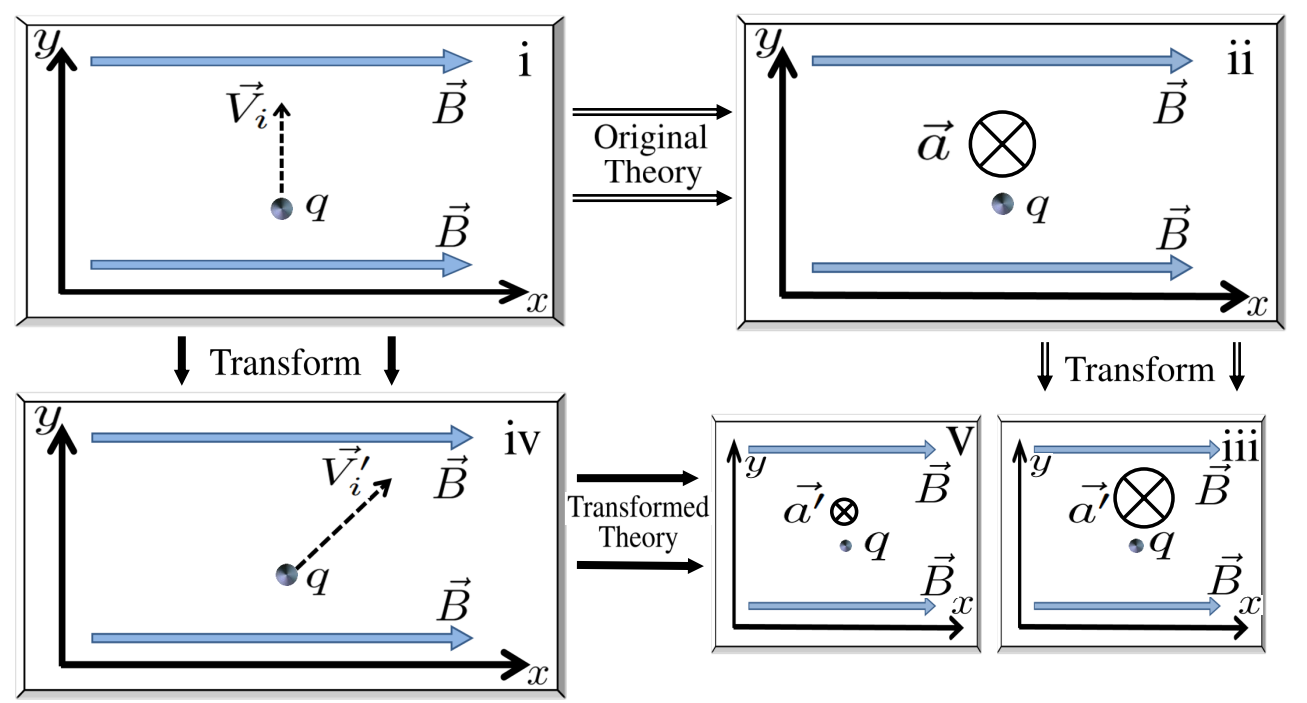

Figure 3. Illustration of effective particle-rotation symmetry violation in a sample system.

Workers in the lab will then know if the system is particle-Lorentz invariant or not by performing their experiment, then rotating it, then comparing the results. If the acceleration is different in the rotated system, particle-Lorentz invariance is broken. In our discussion of the SME to follow, it will be large-scale fields called coefficients for Lorentz violation, perhaps associated with spontaneous breaking of Lorentz symmetry at the Planck scale, which will play the role of $\vec{B}$ and be sought in this manner. We also note in passing that if an undetected large-scale "conventional" field existed in the lab, it could also be detected in this way, an idea that has been applied to efforts to detect spacetime torsion [15] and gravitomagnetic effects in the lab [16]. Additional examples similar to the one presented here can be found in Refs. [10,17].

\section{2. $C P T$}

The role of CPT symmetry and CPT violation can also be illustrated in the above example. Under $\mathrm{CPT}, q$ and $\vec{A}$ change signs, but nothing else. Hence, the theory is invariant. A subtlety is that although $\vec{A}$ changes sign, $\vec{B}$ does not. Figure 4 illustrates the usual procedure for seeing that our example is CPT invariant.

If we again treat $\vec{A}$ as a nontransforming background while workers in the lab build the CPT transformed version of their device, then $\vec{A}$ will not change under CPT and the second term in the theory will change sign for the laboratory CPT transformed system. Hence, the presence of a background $\vec{A}$ leads to effective CPT violation as well as Lorentz violation. The effective CPT violation induced by $\vec{A}$ is illustrated in Figure 5 .

The violation of both CPT and Lorentz symmetry is a feature shared quite generally by backgrounds with odd numbers of indices. For a somewhat more technical review of the connection between CPT and Lorentz symmety, see the contribution to this issue by Lehnert [18]. For simple examples having backgrounds with even numbers of indices, see Refs. [10,17]. 


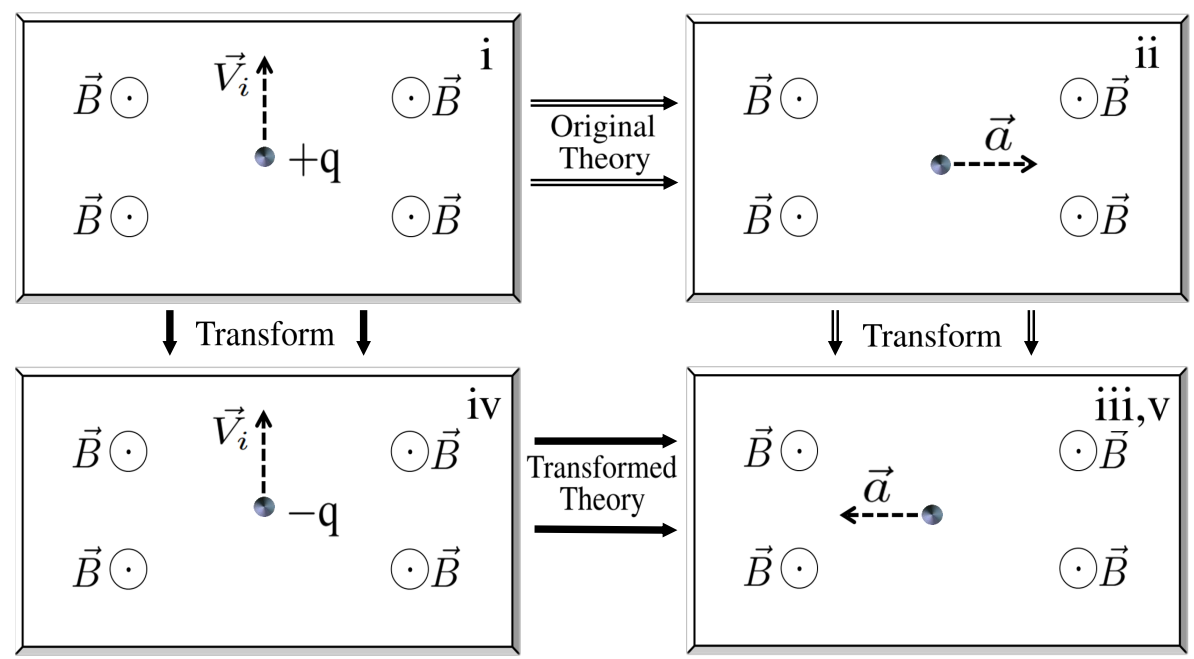

Figure 4. Illustration of CPT symmetry in a sample system.

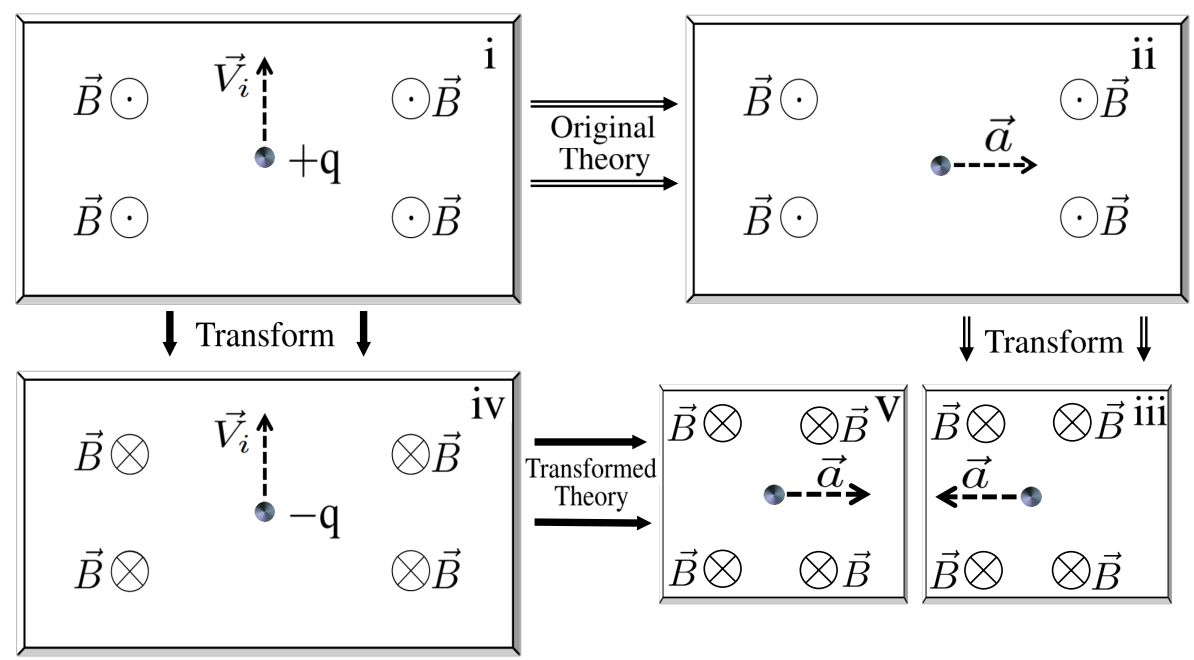

Figure 5. Illustration of an effective CPT-symmetry violation in a sample system.

\section{The SME}

In this section, we present the SME action with a focus on the sectors relevant for the discussion and development of the gravitational results considered in the rest of this work. For a similar review focused on neutrinos, see the contribution to this issue by Díaz [19]. For some review of other sectors, see Ref. [10]. Gravitational phenomenology in the SME can be thought of as originating in two places: the pure-gravity sector, which describes the dynamics of the gravitational field itself, and gravitational couplings in the other sectors. We consider first the gravitationally coupled fermion section, and then the pure-gravity sector below.

\subsection{Gravitationally Coupled Matter}

The implications of gravitational couplings in the minimal fermion sector have been studied considerably. Here, we review the structure of this limit, and use it as an example of the SME structure more generally. The Lagrange density for the minimal fermion sector with gravitational couplings reads [5]:

$$
\mathcal{L}_{\psi-g}=\frac{1}{2} i e e^{\mu}{ }_{a} \bar{\psi} \Gamma^{a} \stackrel{\leftrightarrow}{D_{\mu}} \psi-e \bar{\psi} M \psi
$$


where

$$
\begin{aligned}
\Gamma^{a} & \equiv \gamma^{a}-c_{\mu \nu} e^{\nu a} e^{\mu}{ }_{b} \gamma^{b}-d_{\mu \nu} e^{\nu a} e^{\mu}{ }_{b} \gamma_{5} \gamma^{b}-e_{\mu} e^{\mu a}-i f_{\mu} e^{\mu a} \gamma_{5}-\frac{1}{2} g_{\lambda \mu \nu} e^{v a} e^{\lambda}{ }_{b} e^{\mu}{ }_{c} \sigma^{b c} \\
M & \equiv m+a_{\mu} e^{\mu}{ }_{a} \gamma^{a}+b_{\mu} e^{\mu}{ }_{a} \gamma_{5} \gamma^{a}+\frac{1}{2} H_{\mu \nu} e^{\mu}{ }_{a} e^{v}{ }_{b} \sigma^{a b}
\end{aligned}
$$

Here, $\psi$ is the fermion field, $D_{\mu}$ is the covariant derivative for gravity as well as $U(1)$, and $e_{\mu}{ }^{a}$ is the vierbein, which, along with its determinant $e$ and the covariant derivative, provides the couplings to gravity by linking each point on spacetime manifold with a Minkowski tangent space. The Dirac gamma matricies are denoted $\gamma^{a}$, and $\gamma_{5}$ and $\sigma^{a b}$ denote standard combinations there of [20]. The objects $a \ldots H$ are coefficient fields for Lorentz violation, which, in general, are different for different types of particles. The interpretation of the coefficient fields is developed further below. The field-theoretic and geometric structure suggested by the objects introduced above is rich, but a full review of this would take us too far afield from the phenomenological development, which is the primary subject of this work. For more on these issues, see Ref. [10] and references therein.

Before developing the gravitational implications of (3), we point out several features of (3) with the goal of generating a broader understanding of the SME structure. First, note that the form of the Minkowski-spacetime SME [4] can be recovered in the limit $e_{\mu}{ }^{a} \rightarrow \delta_{\mu}^{a}$, and the standard Lorentz-invariant Dirac Lagrange density can be recovered with the additional restriction $a \ldots H \rightarrow 0$. This last limit reflects a key feature of the SME structure: it contains known physics and can be thought of as providing an expansion about known physics in Lorentz-violating operators. The action (3) is known as the minimal SME limit as it contains operators of dimension 3 and 4 that are power-counting renormalizable. For a basic introduction to the idea of operator dimension, see Ref. [21]. The expansion about known physics can be continued beyond dimension 3 and 4 and the full series of Lorentz violating operators in the fermion sector has been studied [8] in the Minkowski-spacetime limit, as have other sectors [6,7]. Consideration of higher dimension operators in the pure-gravity sector has also begun $[14,22-24]$ and the key points are reviewed in the next subsection.

The content of the coefficient fields $a \ldots H$ is in principle determined by how Lorentz symmetry is broken, though in practice we proceed under general considerations without specializing to a particular model of Lorentz-symmetry breaking. The ways in which Lorentz-symmetry can be broken can be divided into two classes: explicit and spontaneous. Explicit breaking involves coefficients for Lorentz violation that are externally prescribed properties of the spacetime. When Lorentz violation arises spontaneously, the underlying theory is Lorentz-invariant, but the low-energy solutions violate Lorentz symmetry through a process of spontaneous symmetry-breaking analogous to electroweak symmetry breaking in the SM. In flat-spacetime studies, the coefficient fields are typically taken as constant coefficients, satisfying, for example, $\partial_{\alpha} c_{\mu v}=0$. This can be thought of as either a particular explicit choice or as a search for a constant vacuum expectation value given to the field via spontaneous breaking. The consideration of constant coefficients maintains energy-momentum conservation and could be thought of as a leading contribution to more complex spacetime dependence. In curved spacetime, constant coefficients fields are typically not compatible with a theory of gravity based on Riemannian geometry $[5,25,26]$ and specialization to spontaneous breaking is chosen for most SME phenomenology. Here, the coefficient fields can be thought of as involving a constant vacuum value plus a fluctuation about the vacuum, for example $c_{\mu v}=\bar{c}_{\mu v}+\stackrel{\leftarrow}{c}_{\mu v}$. In the asymptotically flat studies considered here, the vacuum values such as $\bar{c}_{\mu v}$ are taken as a constant and can be identified with the coefficients for Lorentz violation explored in flat-spacetime studies. Hence, we refer to the vacuum values as the coefficients for Lorentz violation. Under fairly general assumptions in the work considered here, the fluctuations can be integrated out prior to the development of phenomenology [27-29]. An approach that considers non-constant coefficient fields under a different set of assumptions has also now been developed [30].

For most gravitational experiments, the classical, post-Newtonian implications of the relativistic quantum field theory (3) is what is relevant. As a result, tools corresponding to (3) including the 
relativistic Hamiltonian, the nonrelativistic Hamiltonian, and the classical Lagrangian have been developed to third post Newtonian order [27]. Note that the matter theory impacts the structure of the spacetime since the metric is derived from the matter theory involving coefficients for Lorentz violation associated with the source, as well as the motion of test particles in that spacetime, which involves coefficients for Lorentz violation associated with the test particle. As an example of the tools that result, in the Newtonian limit, the equation of motion for a particle in an Earth-based laboratory associated with spin-independent effects takes the form

$$
F_{j}=m_{j k} \ddot{x}_{k}
$$

where

$$
m_{j k}=m^{\mathrm{T}}\left(1+\left(\bar{c}^{\mathrm{T}}\right)_{t t}\right) \delta_{j k}+2 m^{\mathrm{T}}\left(\bar{c}^{\mathrm{T}}\right)_{(j k)}
$$

and the vertical component of the force is

$$
F_{z}=-m^{\mathrm{T}} g\left[1+\frac{2 \alpha}{m^{\mathrm{T}}}\left(\bar{a}_{\mathrm{eff}}^{\mathrm{T}}\right)_{t}+\frac{2 \alpha}{m^{\mathrm{S}}}\left(\bar{a}_{\mathrm{eff}}^{\mathrm{S}}\right)_{t}+\left(\bar{c}^{\mathrm{T}}\right)_{t t}+\left(\bar{c}^{\mathrm{S}}\right)_{t t}\right]
$$

Here, $\alpha$ is a constant that characterizes couplings in the underlying theory of spontaneous breaking, $m^{\mathrm{T}}$ and $m^{\mathrm{S}}$ are masses of the test and source bodies, respectively, and superscripts $\mathrm{T}$ and $\mathrm{S}$ refer to the coefficients associated with the test and source bodies, respectively. The combination $a_{\mu}-m e_{\mu}$ is denoted $\left(a_{\mathrm{eff}}\right)_{\mu}$. Note that in addition to generating the annual and sidereal variations associated with boost and rotation invariance in the lab, violations of the Weak Equivalence Principle (WEP) are induced by the particle-species dependence in this result.

\subsection{Pure Gravity}

Maxwell's electrodynamics is famously a linear theory, meaning that if one takes the potential $A_{\mu}$ as the fundamental object, the field equations are linear in $A_{\mu}$. This permits linear superposition as a convenient method of constructing solutions. General Relativity is a nonlinear theory of gravity based on the curvature of spacetime encoded in the spacetime metric $g_{\mu v}$, which can be written in terms of the Minkowski metric $\eta_{\mu v}$ plus an object $h_{\mu v}$, typically called the metric perturbation. By analogy with electrodynamics, if $h_{\mu v}$ is taken as the fundamental object, the GR field equations are nonlinear in $h_{\mu v}$, hence nonlinear Lorentz-violating corrections to GR might be expected in an SME expansion about the GR action. However, it should also be noted that such nonlinearities are negligibly small in practice in large classes of experiments where the spacetime can be considered asymptotically Minkowski and $h_{\mu \nu}$ can be treated as a small correction.

Progress toward phenomenology in the pure-gravity sector of the SME began with the development [5] and exploration [29] of its minimal limit. While the construction at the level of the action developed in these works includes the full nonlinear theory of gravity, the development of phenomenological tools is specialized to the linearized-gravity limit [29]. More recently, exploration of the nonminimal pure-gravity sector has begun [14,22-24], including consideration of some terms associated with the nonlinearities of gravity [24]. To provide a maximally coherent treatment of phenomenology and experiment, we consider first the full SME expansion under the restriction of linearized gravity, then offer some comments about current proposals to explore effects in nonlinear gravity.

The generic form of the Lagrange density in the linearized limit including both the Lorentz-violating and Lorentz-invariant contributions can be written: [23],

$$
\mathcal{L}_{\mathcal{K}^{(d)}}=\frac{1}{4} h_{\mu v} \widehat{\mathcal{K}}^{(d) \mu v \rho \sigma} h_{\rho \sigma}
$$

where the operator 


$$
\widehat{\mathcal{K}}^{(d) \mu \nu \rho \sigma}=\mathcal{K}^{(d) \mu \nu \rho \sigma \varepsilon_{1} \varepsilon_{2} \ldots \varepsilon_{d-2}} \partial_{\varepsilon_{1}} \partial_{\varepsilon_{2}} \ldots \partial_{\varepsilon_{d-2}}
$$

has mass dimension $d \geq 2$, and the coefficients $\mathcal{K}^{(d) \mu \nu \rho \sigma \varepsilon_{1} \varepsilon_{2} \ldots \varepsilon_{d-2}}$ are taken as constant and small. An exploration of the operator (10) by decomposition into irreducible parts reveals that 14 classes of operators are involved. Of these, three classes, written as follows,

$$
\widehat{\mathcal{K}}^{(d) \mu v \rho \sigma} \supset \widehat{s}^{\mu v \rho \sigma}+\widehat{q}^{\mu v \rho \sigma}+\widehat{k}^{\mu v \rho \sigma}
$$

are consistent with the usual gauge invariance of GR. The term

$$
\widehat{s}^{\mu \nu \rho \sigma}=\sum_{\text {even } d \geq 4} s^{(d) \mu \rho \circ v \sigma 0^{d-3}}
$$

contains operators at each even dimension greater than or equal to four, hence it contains a minimal contribution. Here, a circle denotes a contraction with a partial derivative. The contribution

$$
\widehat{q}^{\mu \nu \rho \sigma}=\sum_{\text {odd } d \geq 5} q^{(d) \mu \rho \circ v \circ \sigma \circ 0^{d-4}}
$$

involves operators of odd dimension greater than or equal to five, and

$$
\widehat{k}^{\mu v \rho \sigma}=\sum_{\operatorname{even} d \geq 6} k^{(d) \mu \circ \nu \circ \rho \circ \sigma 0^{d-5}}
$$

contributes operators of even dimension greater than or equal to six. For further discussion of their properties, see [23]. Study to date has been focused on these operators, which are associated with spontaneous Lorentz violation.

Operators $\widehat{q}^{\mu \nu \rho \sigma}$ and $\widehat{k}^{\mu v \rho \sigma}$ are associated with birefringence of gravitational waves. Constraints on the dimension 5 and 6 coefficients they contain [23] have been placed using the initial direct observation of gravitational waves by LIGO [31]. Constraints based on the dispersion triggered by isotropic combinations of coefficients at dimentions 4, 5, and 6 have also been attained [32] based on LIGO observations of merger events [31,33]. We note in passing that LIGO has also been used to achieve photon-sector sensitivities by interpreting a suitable aspect of the 2006-2007 LIGO data as providing a Michelson-Morley-type test [34]. Matter-sector implications have also been noted [35].

The nature of $\widehat{s}^{\mu v \rho \sigma}$ can be elucidated by introducing a dual operator as follows: $\widehat{s}^{\mu \rho v \sigma}=-\epsilon^{\mu \rho \alpha \kappa} \epsilon^{\alpha \nu \sigma \beta \lambda} \widehat{\bar{s}}_{\kappa \lambda} \partial_{\alpha} \partial_{\beta}$. In terms of the dual, the Lagrange density can be written,

$$
\mathcal{L}=\frac{1}{4} \epsilon^{\mu \rho \alpha \kappa} \epsilon^{v \sigma \beta \lambda} h_{\mu v}\left(\eta_{\kappa \lambda}-\widehat{\bar{s}}_{\kappa \lambda}\right) \partial_{\alpha} \partial_{\beta} h_{\rho \sigma},+\frac{1}{4} h_{\mu v}\left(\widehat{q}^{\mu \rho v \sigma}+\widehat{k}^{\mu v \rho \sigma}\right) h_{\rho \sigma}
$$

where $\eta_{\kappa \lambda}$ in the first term is the appropriate limit of the standard Einstein-Hilbert contribution. The use of the dual operator reveals that $\widehat{s}^{\mu \rho v \sigma}$ acts as a momentum-dependent perturbation to the Minkowski metric. As in the case of the general operator introduced in Equation (11), $\hat{\bar{s}}_{\mu v}$ can be expanded in terms of coefficients for Lorentz violation at each dimension $d$, which are taken as constant and small in most studies, and an appropriate number of derivatives. Explicitly,

$$
\hat{\bar{s}}_{\mu v} \equiv \sum_{\text {even } d \geq 4}\left(\bar{s}^{(d)}\right)_{\mu \nu}{ }^{\alpha_{1} \ldots \alpha_{d-4}} \partial_{\alpha_{1}} \ldots \partial_{\alpha_{d-4}}
$$

Here, $\bar{s}_{\mu \nu}^{(4)} \equiv \bar{s}_{\mu \nu}$ is the coefficient studied in numerous minimal SME investigations as noted in the next section. An additional coefficient coupling to the Weyl tensor, $t^{\mu \nu \rho \sigma}$ was considered in the original investigation of the minimal gravity sector [5], but phenomenological implications stemming from this coefficient have not been found [36]. Additionally, elements of the sum (16) at dimension 4, 6, and 8 
have been sought via gravitational Čerenkov radiation. We summarize this method and expand on the result in Section 5 of this work where constraints on the $d=10$ coefficients are achieved.

A combination of $\widehat{s}^{\mu \rho v \sigma}$ and $\widehat{k}^{\mu \rho v \sigma}$ coefficients at $d=6$ named $\left(\bar{k}_{\text {eff }}\right)_{j k l m}$ have been identified [22] as contributing to short-range gravity experiments via the modified Poisson equation

$$
-\nabla^{2} U=4 \pi G_{N} \rho+\left(\bar{k}_{\text {eff }}\right)_{j k l m} \partial_{j} \partial_{k} \partial_{l} \partial_{m} U
$$

Here $U(\vec{r})$ is the modified Newtonian gravitational potential, and $G_{N}$ is Newton's constant.

Initial studies of Lorentz violation in nonlinear gravity have also been performed [24] that focus on the $d=8$ (12 index) coefficient field that couples to three Riemann curvature tensors in the action. The phenomenology of a subset of the vacuum values associated with this coefficient field denoted $K_{j k}$ has been established.

\section{Gravitational Tests: Existing Results and Proposals}

A large amount of experimental and observational work has been done based on the theory reviewed above, and numerous proposals exist to extend and improve these results. In Table 1, we list systems that have been used to achieve constraints on coefficients for Lorentz violation. The first column identifies the system; the second, the coefficients constrained; the third, references to the associated phenomenological proposals; and the fourth, references to the work in which the constraints were achieved. Note that in some cases there is overlap between the last two columns. In some cases, initial constraints on coefficients for Lorentz violation are achieved in the paper proposing the work based on a reinterpretation of published information. In other cases, the work is essentially proposed in the experimental paper. An asterisk $(*)$ denotes coefficients constrained in Section 5 of this work. In addition to information provided in the papers cited, the constraints achieved in each test are summarized in [9].

Table 1. Gravitational tests constraining Standard-Model Extension (SME) coefficients

\begin{tabular}{|c|c|c|c|}
\hline System & Coefficients & Proposal & Constraints \\
\hline gravitational Čerenkov radiation & $\bar{s}_{\mu \nu}^{(4)}, \bar{s}_{\mu \nu \alpha_{1} \alpha_{2}}^{(6)}, \bar{s}_{\mu \nu \alpha_{1} \alpha_{2} \alpha_{3} \alpha_{4}}, \bar{s}_{\mu v \alpha_{1} \alpha_{2} \alpha_{3} \alpha_{4} \alpha_{5} \alpha_{6}}^{(10)}$ & [14] & {$[14][*]$} \\
\hline $\begin{array}{l}\text { superconducting gravimeters } \\
\text { short-range gravity devices }\end{array}$ & $\begin{array}{l}\left(\bar{a}_{\text {eff }}\right)_{\mu}, \bar{c}_{\mu v}, \bar{s}_{\mu v}^{(4)} \\
\left(\bar{k}_{\text {eff }}\right)_{j k l m}\left(\bar{a}_{\text {eff }}\right)_{\mu}\end{array}$ & $\begin{array}{c}{[27,29]} \\
{[22,27,29]}\end{array}$ & $\begin{array}{c}{[37]} \\
{[38-42]}\end{array}$ \\
\hline gravitational-wave interferometers & $\bar{s}_{\mu \nu}^{(4)}, \bar{s}_{\mu \nu \alpha_{1} \alpha_{2}}^{(6)} q^{(5) \mu \rho \alpha \nu \beta \sigma \gamma}, k^{(6) \mu \alpha \nu \beta \rho \gamma \sigma \delta}$ & [23] & {$[23,32]$} \\
\hline lunar laser ranging & $\bar{s}_{\mu \nu}^{(4)}$ & [29] & {$[43,44]$} \\
\hline binary-pulsar observations & $\bar{s}_{\mu \nu}^{(4)}$ & [29] & [45-47] \\
\hline planetary ephemerides & $\left(\bar{a}_{\text {eff }}\right)_{\mu}, \bar{s}_{\mu v}^{(4)}$ & [48] & [48] \\
\hline gravity probe B & $\bar{s}_{\mu v}^{(4)}$ & [29] & [49] \\
\hline bound kinetic energy WEP & $\left(\bar{a}_{\text {eff }}\right)_{\mu}, \bar{c}_{\mu v}$ & {$[27,50]$} & [50] \\
\hline atom interferometers & $\left(\bar{a}_{\mathrm{eff}}\right)_{\mu}, \bar{c}_{\mu v}, \bar{s}_{\mu \nu}^{(4)}$ & {$[27,29]$} & [51-53] \\
\hline comagnetometry & $\left(\bar{a}_{\mathrm{eff}}\right)_{\mu}, \overline{\mathrm{s}}_{\mu \nu}^{(4)}$ & [16] & [16] \\
\hline perihelion precession & $\left(\bar{a}_{\text {eff }}\right)_{\mu}, \bar{c}_{\mu v}$ & [27] & [27] \\
\hline equivalence-principle pendulum & $\left(\bar{a}_{\text {eff }}\right)_{\mu}$ & [28] & [28] \\
\hline Solar-spin precession & $\bar{s}_{\mu \nu}^{(4)}$ & [29] & [29] \\
\hline
\end{tabular}

In Table 2, we highlight some of the cases in which existing or planned experiments could provide improvement over published limits. Here, improvements could involve extending the maximum reach for a given coefficient, generating sensitivities to new linear combinations of coefficients that lead to either more independent constraints or a discovery, or the generation of cleaner constraints that involve fewer assumptions. The first three columns are the same as in Table 1. The last column lists references to some existing and planned experiments associated with the given system that hold 
promise for improving sensitivities to the coefficients listed. Note that no such table can be exhaustive. The list provided here is merely intended to highlight the breath of possibilities.

Table 2. Some systems that could improve upon existing sensitivities.

\begin{tabular}{|c|c|c|c|}
\hline System & Coefficients & Proposal & Experiments \\
\hline atom interferometer & $\left(\bar{a}_{\mathrm{eff}}\right)_{\mu}, \bar{c}_{\mu \nu}, \bar{s}_{\mu \nu}^{(4)}$ & [27] & [54] \\
\hline ring-laser gyroscopes & $\bar{s}_{\mu \nu}^{(4)}$ & {$[55,56]$} & [57] \\
\hline torsion pendula & $\left(\bar{a}_{\text {eff }}\right)_{\mu}, \bar{c}_{\mu v}$ & [27] & [58] \\
\hline binary pulsars & $\left(\bar{a}_{\text {eff }}\right)_{\mu}, \bar{c}_{\mu \nu}, \bar{s}_{\mu \nu}^{(4)} K_{j k}$ & {$[24,59]$} & [60] \\
\hline short-range gravity & $K_{j k}$ & [24] & {$[38,39,42]$} \\
\hline gravitational-wave detectors & $s^{(d) \mu \rho \alpha v \sigma \beta \ldots}, k^{(d) \mu \alpha v \beta \rho \gamma \sigma \delta \ldots \ldots}, q^{(d) \mu \rho \alpha v \beta \sigma \gamma \ldots}$ & {$[23]$} & {$[31,33]$} \\
\hline space-based WEP tests & $\left(\bar{a}_{\mathrm{eff}}\right)_{\mu}, \bar{c}_{\mu \nu}$ & {$[29,61]$} & [61-64] \\
\hline antimatter gravity & $\left(\bar{a}_{\mathrm{eff}}\right)_{\mu}, \bar{c}_{\mu v}$ & {$[27,65]$} & [66-69] \\
\hline charged matter WEP & $\left(\bar{a}_{\mathrm{eff}}\right)_{\mu}$ & [27] & [70] \\
\hline muonium free fall & $\left(\bar{a}_{\mathrm{eff}}\right)_{\mu}, \bar{c}_{\mu \nu}$ & [27] & [71] \\
\hline light bending & $\left(\bar{a}_{\mathrm{eff}}\right)_{\mu}, \bar{c}_{\mu \nu}, \bar{s}_{\mu \nu}^{(4)}$ & [72] & [73] \\
\hline time-delay \& Doppler tests & $\bar{s}_{\mu \nu}^{(4)}$ & {$[27,74]$} & [75-77] \\
\hline
\end{tabular}

\section{Gravitational Čerenkov}

In this section, we obtain and present the first limits on the dimension 10 coefficients contained within $\hat{\bar{s}}^{\alpha \beta}$ by following the methods of Ref. [14]. Prior to presenting the results, we summarize some of the key ideas used.

As is well known, when charged particles exceed the phase speed of light in media, the Čerenkov radiation of photons results. In the presence of the Lorentz violation in the SME, the vacuum exhibits many of the properties of the medium, and vacuum Čerenkov radiation becomes kinematically allowed. This feature extends to the case of gravitational waves, where, in the presence of certain coefficients for Lorentz violation, particles may exceed the speed of gravity and emit Čerenkov gravitons $[14,78]$. This feature is possible for massive particles as well as photons, and, in the presence of suitable SME coefficients, can be both anisotropic and momentum dependent [14].

The idea of vacuum gravitational Čerenkov radiation can be used to constrain coefficients for Lorentz violation through consideration of high-energy cosmic rays. The fact that these rays arrive at Earth from great distances with high energy limits the amount of energy they could have radiated to gravitational waves, and hence limits the coefficients for Lorentz violation involved.

To begin our analysis, we note that the dispersion relation associated with $\hat{\bar{s}}^{\alpha \beta}$ can be written

$$
l_{0}^{2}=\vec{l}^{2}+\hat{\bar{s}}^{\alpha \beta} l_{\alpha} l_{\beta}
$$

where $l_{\mu}$ is the graviton momentum. The form of Equation (18) implies that it is convenient to introduce an effective momentum-dependent vacuum index of refraction for gravity,

$$
n(\vec{l})=\sqrt{1-\hat{\bar{s}}^{\alpha \beta} \hat{l}_{\alpha} \hat{l}_{\beta}}
$$

Standard decay-rate and energy-loss equations can then be used to find the rate of energy loss to gravitational waves by a faster-than-gravity particle, which can be written

$$
\frac{d E}{d t}=-\frac{1}{8|\vec{p}| \sqrt{m_{w}^{2}+\vec{p}^{2}}} \int \frac{d^{3} l}{(2 \pi)^{2}|\vec{l}|}|\mathcal{M}|^{2} \delta\left(\cos \theta-\cos \theta_{C}\right)
$$


Here, $p_{\mu}$ is the particle momentum, $m_{w}$ is its mass, $\mathcal{M}$ is a matrix element from quantum field theory, the angle between $\vec{p}$ and $\vec{l}$ is $\theta$, and $\theta_{C}$ is a generalized Čerenkov angle that takes the form

$$
\cos \theta_{C}=\frac{\sqrt{m_{w}^{2}+\vec{p}^{2}}}{|\vec{p}|} \frac{1}{n(|\vec{l}|)}+\frac{|\vec{l}|}{2|\vec{p}|}\left(1-\frac{1}{[n(|\vec{l}|)]^{2}}\right)
$$

Inserting the matrix element associated with the given radiating particle and integrating over graviton momentum yields an explicit form for the power loss to radiated gravitons. The result is the same for each type of particle considered (photons, scalars, and fermions) up to a dimension $d$ dependent factor. This result can then be integrated to get a relation between the initial energy $E_{i}$ at the start of the particle's trip, the final energy $E_{f}$ at detection, and the time of flight $t$ :

$$
t=\frac{\mathcal{F}^{w}(d)}{G_{N}\left(s^{(d)}\right)^{2}}\left(\frac{1}{E_{f}^{2 d-5}}-\frac{1}{E_{i}^{2 d-5}}\right)
$$

Here $\mathcal{F}^{w}(d)$ is the species-dependent factor. In the analysis to follow, we consider fermions for which

$$
\mathcal{F}^{\psi}(d)=\frac{(d-2)(d-3)(2 d-3)}{4\left(2 d^{2}-7 d+9\right)}
$$

The dependence on Lorentz violation in Equation (22) is through the combination

$$
s^{(d)}(\hat{p}) \equiv\left(\bar{s}^{(d)}\right)^{\mu \nu \alpha_{1} \ldots \alpha_{d-4}} \hat{p}_{\mu} \hat{p}_{v} \hat{p}_{\alpha_{1}} \ldots \hat{p}_{\alpha_{d-4}}
$$

which can also be expressed in terms of spherical harmonics and spherical coefficients for Lorentz violation as

$$
s^{(d)}(\hat{p})=\sum_{j m} Y_{j m}(\hat{p}) \bar{s}_{j m}^{(d)}
$$

Constraints on the coefficients $s^{(d)}$ are achieved via high-energy cosmic ray observations from the following projects: Akeno Giant Air Shower Array (AGASA) [79,80], Fly's Eye [81], Haverah Park [79,82], HiRes [83], Pierre Auger [84], Sydney University Giant Air-shower Recorder (SUGAR) [79,85], Telescope Array [86], Volcano Ranch [79,87], and Yakutsk [79,88]. For details on the events used, see Table 1 in Ref. [14]. Extracting a constraint requires knowledge of the initial and final energy and the distance traveled, $L$. To generate conservative constraints, we take $E_{i}=\infty$ and solve for the coefficients

$$
s^{(d)}(\hat{p})<\sqrt{\frac{\mathcal{F}^{w}(d)}{G_{N} E_{f}^{2 d-5} L}}
$$

To generate a value for $L$ we consider the likely origin of the highest energy cosmic rays, which are believed to be nuclei originating from an active galactic nucleus nearby. For a conservative and definite number, we take $L \approx 10 \mathrm{Mpc}$. Finally, we need the final energy as the particles arrive at Earth, which is based on the observed energy. Again proceeding toward conservative constraints, we assume that a partonic fermion carrying $10 \%$ of the proton energy in an iron nucleus is the radiator of gravitons. Hence, we take $E_{f}$ as $1 / 560$ of the observed energy.

One observation typically yields a one-sided constraint on a combination of coefficients for Lorentz violation, since gravitational Čerenkov radiation is only possible for particles moving faster than the speed of gravity. However, with the exception of the isotropic coefficients, two-sided constraints are achieved using cosmic rays originating from multiple places on the sky. In Ref. [14], a series of six models were constrained. The models included three isotropic models containing the isotropic coefficient at $d=4, d=6$, and $d=8$, respectively, and three anisotropic models containing the rest of the coefficients at each dimension. The numerical constraints were obtained from the energy and direction of origin for observed cosmic rays using a linear programming scheme (for details, see [89]). 
Here, we perform the same operations on the same data for two more models: an isotropic $d=10$ model and an anisotropic $d=10$ model. The constraints that result, which are the first on $d=10$ coefficients in the gravity sector, appear in Table 3.

Table 3. Conservative constraints on coefficients $\bar{s}_{j m}^{(10)}$ in $\mathrm{GeV}^{-6}$.

\begin{tabular}{|c|c|c|c|c|c|c|c|}
\hline$j$ & Lower Bound & Coeff. & Upper Bound & $j$ & Lower Bound & Coeff. & Upper Bound \\
\hline 0 & & $\bar{s}_{00}^{(10)}$ & $<2 \times 10^{-66}$ & \multirow{13}{*}{6} & $-2 \times 10^{-61}<$ & $\bar{s}_{60}^{(10)}$ & $<2 \times 10^{-61}$ \\
\hline & $-1 \times 10^{-61}<$ & $\bar{s}_{10}^{(10)}$ & $<2 \times 10^{-61}$ & & $-1 \times 10^{-61}<$ & $\operatorname{Re} \bar{s}_{61}^{(10)}$ & $<1 \times 10^{-61}$ \\
\hline 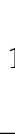 & $-1 \times 10^{-61}<$ & $\operatorname{Re} \bar{s}_{11}^{(10)}$ & $<1 \times 10^{-61}$ & & $-1 \times 10^{-61}<$ & $\operatorname{Im} \bar{s}_{61}^{(10)}$ & $<1 \times 10^{-61}$ \\
\hline & $-1 \times 10^{-61}<$ & $\operatorname{Im} \bar{s}_{11}^{(10)}$ & $<1 \times 10^{-61}$ & & $-8 \times 10^{-62}<$ & $\operatorname{Re} \bar{s}_{62}^{(10)}$ & $<2 \times 10^{-61}$ \\
\hline \multirow{5}{*}{2} & $-2 \times 10^{-61}<$ & $\bar{s}_{20}^{(10)}$ & $<1 \times 10^{-61}$ & & $-1 \times 10^{-61}<$ & $\operatorname{Im} \bar{s}_{62}^{(10)}$ & $<1 \times 10^{-61}$ \\
\hline & $-1 \times 10^{-61}<$ & $\operatorname{Re} \bar{s}_{21}^{(10)}$ & $<1 \times 10^{-61}$ & & $-1 \times 10^{-61}<$ & $\operatorname{Re} \bar{s}_{63}^{(10)}$ & $<9 \times 10^{-62}$ \\
\hline & $-1 \times 10^{-61}<$ & $\operatorname{Im} \bar{s}_{21}^{(10)}$ & $<1 \times 10^{-61}$ & & $-1 \times 10^{-61}<$ & $\operatorname{Im} \bar{s}_{63}^{(10)}$ & $<1 \times 10^{-61}$ \\
\hline & $-1 \times 10^{-61}<$ & $\operatorname{Re} \bar{s}_{22}^{(10)}$ & $<1 \times 10^{-61}$ & & $-1 \times 10^{-61}<$ & $\operatorname{Re} \bar{s}_{64}^{(10)}$ & $<1 \times 10^{-61}$ \\
\hline & $-1 \times 10^{-61}<$ & $\operatorname{Im} \bar{s}_{22}^{(10)}$ & $<1 \times 10^{-61}$ & & $-1 \times 10^{-61}<$ & $\operatorname{Im} \bar{s}_{64}^{(10)}$ & $<1 \times 10^{-61}$ \\
\hline \multirow{7}{*}{3} & $-2 \times 10^{-61}<$ & $\bar{s}_{30}^{(10)}$ & $<2 \times 10^{-61}$ & & $-1 \times 10^{-61}<$ & $\operatorname{Re} \bar{s}_{65}^{(10)}$ & $<1 \times 10^{-61}$ \\
\hline & $-1 \times 10^{-61}<$ & $\operatorname{Re} \bar{s}_{31}^{(10)}$ & $<1 \times 10^{-61}$ & & $-1 \times 10^{-61}<$ & $\operatorname{Im}$ & $<1 \times 10^{-61}$ \\
\hline & $-1 \times 10^{-61}<$ & $\operatorname{Im} \bar{s}_{31}^{(10)}$ & $<1 \times 10^{-61}$ & & $-1 \times 10^{-61}<$ & $\operatorname{Re}$ & $<1 \times 10^{-61}$ \\
\hline & $-1 \times 10^{-61}<$ & $\operatorname{Re} \bar{s}_{32}^{(10)}$ & $<1 \times 10^{-61}$ & & $-1 \times 10^{-61}<$ & $\operatorname{Im} \bar{s}_{66}^{(10)}$ & $<1 \times 10^{-61}$ \\
\hline & $-1 \times 10^{-61}<$ & $\operatorname{Im} \bar{s}_{32}^{(10)}$ & $<1 \times 10^{-61}$ & \multirow{15}{*}{7} & $-2 \times 10^{-61}<$ & $\bar{s}_{70}^{1}$ & $<2 \times 10^{-61}$ \\
\hline & $-1 \times 10^{-61}<$ & $\operatorname{Re} \bar{s}_{33}^{(10)}$ & $<1 \times 10^{-61}$ & & $-9 \times 10^{-62}<$ & & $<1 \times 10^{-61}$ \\
\hline & $-1 \times 10^{-61}<$ & $\operatorname{Im} \bar{s}_{33}^{(10)}$ & $<1 \times 10^{-61}$ & & $-1 \times 10^{-61}<$ & $\operatorname{Im}$ & $<9 \times 10^{-62}$ \\
\hline \multirow{9}{*}{4} & $-2 \times 10^{-61}<$ & $\bar{s}_{40}^{(10)}$ & $<1 \times 10^{-61}$ & & $-1 \times 10^{-61}<$ & $\operatorname{Re} \bar{s}$ & $<9 \times 10^{-62}$ \\
\hline & $-2 \times 10^{-61}<$ & $\operatorname{Re} \bar{s}_{41}^{(10)}$ & $<1 \times 10^{-61}$ & & $-1 \times 10^{-61}<$ & $\mathrm{Im}$ & $<1 \times 10^{-61}$ \\
\hline & $-1 \times 10^{-61}<$ & $\operatorname{Im} \bar{s}_{41}^{(10)}$ & $<1 \times 10^{-61}$ & & $-1 \times 10^{-61}<$ & $\operatorname{Re} \bar{s}$ & $<1 \times 10^{-61}$ \\
\hline & $-1 \times 10^{-61}<$ & $\operatorname{Re} \bar{s}_{42}^{(10)}$ & $<1 \times 10^{-61}$ & & $-1 \times 10^{-61}<$ & $\operatorname{Im} \bar{s}_{73}^{(10)}$ & $<1 \times 10^{-61}$ \\
\hline & $-1 \times 10^{-61}<$ & $\operatorname{Im} \bar{s}_{42}^{(10)}$ & $<1 \times 10^{-61}$ & & $-1 \times 10^{-61}<$ & $\operatorname{Re} \bar{s}_{74}^{(10)}$ & $<1 \times 10^{-61}$ \\
\hline & $-1 \times 10^{-61}<$ & $\operatorname{Re} \bar{s}_{43}^{(10)}$ & $<9 \times 10^{-62}$ & & $-1 \times 10^{-61}<$ & & $<1 \times 10^{-61}$ \\
\hline & $-1 \times 10^{-61}<$ & $\operatorname{Im} \bar{s}_{43}^{(10)}$ & $<1 \times 10^{-61}$ & & $-1 \times 10^{-61}<$ & & $<1 \times 10^{-61}$ \\
\hline & $-1 \times 10^{-61}<$ & $\operatorname{Re} \bar{s}_{44}^{(10)}$ & $<1 \times 10^{-61}$ & & $-1 \times 10^{-61}<$ & $\operatorname{Im} \bar{s}_{75}^{(10)}$ & $<1 \times 10^{-61}$ \\
\hline & $-1 \times 10^{-61}<$ & $\operatorname{Im} \bar{s}_{44}^{(10)}$ & $<1 \times 10^{-61}$ & & $-1 \times 10^{-61}<$ & $\operatorname{Re} \bar{s}_{76}^{(10)}$ & $<1 \times 10^{-61}$ \\
\hline \multirow{20}{*}{5} & $-1 \times 10^{-61}<$ & $\bar{s}_{50}^{(10)}$ & $<2 \times 10^{-61}$ & & $-1 \times 10^{-61}<$ & $\operatorname{Im} \bar{s}_{76}^{(10)}$ & $<1 \times 10^{-61}$ \\
\hline & $-1 \times 10^{-61}<$ & $\operatorname{Re} \bar{s}_{51}^{(10)}$ & $<1 \times 10^{-61}$ & & $-1 \times 10^{-61}<$ & $\operatorname{Re} \bar{s}_{77}^{(10)}$ & $<1 \times 10^{-61}$ \\
\hline & $-1 \times 10^{-61}<$ & $\operatorname{Im} \bar{s}_{51}^{(10)}$ & $<1 \times 10^{-61}$ & & $-2 \times 10^{-61}<$ & $\operatorname{Im} \bar{s}_{77}^{(10)}$ & $<1 \times 10^{-61}$ \\
\hline & $-1 \times 10^{-61}<$ & $\operatorname{Re} \bar{s}_{52}^{(10)}$ & $<1 \times 10^{-61}$ & \multirow{17}{*}{8} & $-2 \times 10^{-61}<$ & $\bar{s}_{80}^{(10}$ & $<2 \times 10^{-61}$ \\
\hline & $-1 \times 10^{-61}<$ & $\operatorname{Im} \bar{s}_{52}^{(10)}$ & $<1 \times 10^{-61}$ & & $-1 \times 10^{-61}<$ & $\operatorname{Re} \bar{s}_{81}^{(10)}$ & $<1 \times 10^{-61}$ \\
\hline & $-1 \times 10^{-61}<$ & $\operatorname{Re} \bar{s}_{53}^{(10)}$ & $<2 \times 10^{-61}$ & & $-1 \times 10^{-61}<$ & $\operatorname{Im}$ & $<1 \times 10^{-61}$ \\
\hline & $-1 \times 10^{-61}<$ & $\operatorname{Im} \bar{s}_{53}^{(10)}$ & $<1 \times 10^{-61}$ & & $-1 \times 10^{-61}<$ & $\operatorname{Re} \bar{s}_{82}^{(10)}$ & $<1 \times 10^{-61}$ \\
\hline & $-1 \times 10^{-61}<$ & $\operatorname{Re} \bar{s}_{54}^{(10)}$ & $<1 \times 10^{-61}$ & & $-1 \times 10^{-61}<$ & $\operatorname{Im}$ & $<1 \times 10^{-61}$ \\
\hline & $-1 \times 10^{-61}<$ & $\operatorname{Im} \bar{s}_{54}^{(10)}$ & $<1 \times 10^{-61}$ & & $-1 \times 10^{-61}<$ & & $<1 \times 10^{-61}$ \\
\hline & $-1 \times 10^{-61}<$ & $\operatorname{Re} \bar{s}_{55}^{(10)}$ & $<9 \times 10^{-62}$ & & $-1 \times 10^{-61}<$ & $\operatorname{Im}$ & $<1 \times 10^{-61}$ \\
\hline & $-1 \times 10^{-61}<$ & $\operatorname{Im} \bar{s}_{55}^{(10)}$ & $<1 \times 10^{-61}$ & & $-1 \times 10^{-61}<$ & $\operatorname{Re}$ & $<2 \times 10^{-61}$ \\
\hline & & & & & $-1 \times 10^{-61}<$ & & $<1 \times 10^{-61}$ \\
\hline & & & & & $-1 \times 10^{-61}<$ & $\operatorname{Re} \bar{s}$ & $<1 \times 10^{-61}$ \\
\hline & & & & & $-1 \times 10^{-61}<$ & & $<1 \times 10^{-61}$ \\
\hline & & & & & $-1 \times 10^{-61}<$ & $\operatorname{Re} \bar{s}_{86}^{(10)}$ & $<2 \times 10^{-61}$ \\
\hline & & & & & $-1 \times 10^{-61}<$ & & $<1 \times 10^{-61}$ \\
\hline & & & & & $-1 \times 10^{-61}<$ & $\operatorname{Re} \bar{s}_{87}^{(10)}$ & $<1 \times 10^{-61}$ \\
\hline & & & & & $-1 \times 10^{-61}<$ & $\operatorname{Im} \bar{s}_{87}^{(10)}$ & $<1 \times 10^{-61}$ \\
\hline & & & & & $-1 \times 10^{-61}<$ & $\operatorname{Re} \bar{s}_{88}^{(10)}$ & $<1 \times 10^{-61}$ \\
\hline & & & & & $-1 \times 10^{-61}<$ & $\operatorname{Im} \bar{s}_{88}^{(10)}$ & $<1 \times 10^{-61}$ \\
\hline
\end{tabular}




\section{Conclusions}

This work summarizes and expands upon gravity-related tests of Lorentz symmetry in the SME. We begin in Section 2 by providing some examples of symmetry and symmetry violation that highlight CPT and Lorentz violation conceptually and provide context for the SME structure reviewed in Section 3. The gravitationally coupled fermion sector and the pure gravity sector are presented as generic examples of the SME construction to provide context for the phenomenology. Existing and proposed tests are summarized in Section 4. Section 5 provides some more detail on one recent test that achieved tight first constraints on dimension 6 and 8 coefficients in the gravity sector through the analysis of gravitational Čerenkov radiation effects on cosmic rays. Following review of that work, 81 tight new constraints on dimension 10 coefficients are achieved following the same methods. Both the new results achieved and the existing proposals for expansion discussed here highlight the bright future for the continued expansion of tests of Lorentz invariance in gravitational experiments.

Conflicts of Interest: The author declares no conflict of interest. The founding sponsors had no role in the design of the study; in the collection, analyses, or interpretation of data; in the writing of the manuscript, and in the decision to publish the results.

\section{References}

1. Kostelecký, V.A.; Samuel, S. Spontaneous breaking of Lorentz symmetry in string theory. Phys. Rev. D $1989,39,683$.

2. Kostelecký, V.A.; Potting, R. CPT and strings. Nucl. Phys. B 1991, 359, 545.

3. Colladay, D.; Kostelecký, V.A. CPT violation and the standard model. Phys. Rev. D 1997, 55, 6760.

4. Colladay, D.; Kostelecký, V.A. Lorentz-violating extension of the standard model. Phys. Rev. D 1998, $58,116002$.

5. Kostelecký, V.A. Gravity, Lorentz violation, and the standard model. Phys. Rev. D 2004, 69, 105009.

6. Kostelecký, V.A.; Mewes, M. Electrodynamics with Lorentz-violating operators of arbitrary dimension. Phys. Rev. D 2009, 80, 015020.

7. Kostelecký, V.A.; Mewes, M. Neutrinos with Lorentz-violating operators of arbitrary dimension. Phys. Rev. D 2012, 85, 096005.

8. Kostelecký, V.A.; Mewes, M. Fermions with Lorentz-violating operators of arbitrary dimension. Phys. Rev. D 2013, 88, 096006.

9. Kostelecký, V.A.; Russell, N. Data tables for Lorentz and CPT violation. Rev. Mod. Phys. 2011, 83, 11.

10. Tasson, J.D. What do we know about Lorentz invariance? Rep. Prog. Phys. 2014, 77, 062901.

11. Liberati, S. Tests of Lorentz invariance: A 2013 update. Class. Quant. Grav. 2013, 30, 133001.

12. Will, C.M. The confrontation between general relativity and experiment. Living Rev. Relativ. 2014, 17, 4.

13. Yunes, N.; Pretorius, F. Fundamental theoretical bias in gravitational wave astrophysics and the parametrized post-Einsteinian framework. Phys. Rev. D 2009, 80, 122003.

14. Kostelecký, V.A.; Tasson, J.D. Constraints on Lorentz violation from gravitational Čerenkov radiation. Phys. Lett. B 2015, 749, 551.

15. Kostelecký, V.A.; Russell, N.; Tasson, J.D. Constraints on torsion from bounds on Lorentz violation. Phys. Rev. Lett. 2008, 100, 111102.

16. Tasson, J.D. Lorentz violation, gravitomagnetism, and intrinsic spin. Phys. Rev. D 2012, 86, 124021.

17. Bertschinger, T.H.; Flowers, N.A.; Tasson, J.D. Observer and particle transformations. In CPT and Lorentz Symmetry; Kostelecký, V.A., Ed.; World Scientific: Singapore, 2014; Volume VI.

18. Lehnert, R. CPT symmetry and its violation. Symmetry 2016, submitted.

19. Díaz, J.S. Testing Lorentz and CPT invariance with neutrinos. Symmetry 2016, 8, 105.

20. Itzykson, C.; Zuber, J.-B. Quantum Field Theory; McGraw-Hill: New York, NY, USA, 1980.

21. Tasson, J.D. Antimatter, Lorentz symmetry, and gravity. Hyperfine Interact. in press.

22. Bailey, Q.G.; Kostelecký, V.A.; Xu, R. Short-range gravity and Lorentz violation Phys. Rev. D 2015, 91, 022006.

23. Kostelecký, V.A.; Mewes, M. Testing local Lorentz invariance with gravitational waves. Phys. Lett. B 2016, 757, 510 .

24. Bailey, Q.G. Anisotropic cubic curvature couplings. Phys. Rev. D 2016, 94, 065029. 
25. Bluhm, R. Explicit versus spontaneous diffeomorphism breaking in gravity. Phys. Rev. D 2015, 91, 065034.

26. Bluhm, R. Spacetime symmetry breaking and Einstein-Maxwell theory. Phys. Rev. D 2015, 92, 085015.

27. Kostelecký, V.A.; Tasson, J.D. Matter-gravity couplings and Lorentz violation. Phys. Rev. D 2011, 83, 016013.

28. Kostelecký, V.A.; Tasson, J.D. Prospects for large relativity violations in matter-gravity couplings. Phys. Rev. Lett. 2009, 102, 010402.

29. Bailey, Q.G.; Kostelecký, V.A. Signals for Lorentz violation in post-Newtonian gravity. Phys. Rev. D 2006, 74, 045001.

30. Lane, C.D. Spacetime variation of Lorentz-violation coefficients at nonrelativistic scale. Phys. Rev. D 2016, 94, 025016.

31. Abbott, B.P.; Abbott, R.; Abbott, T.D.; Abernathy, M.R.; Acernese, F.; Ackley, K.; Adams, C.; Adams, T.; Addesso, P.; Adhikari, R.X.; et al. LIGO scientific collaboration and virgo collaboration. Observation of gravitational waves from a binary black hole merger. Phys. Rev. Lett. 2016, 116, 061102.

32. Yunes, N.; Yagi, K.; Pretorius, F. Theoretical physics implications of the binary black-hole mergers GW150914 and GW151226. Phys. Rev. D 2016, 94, 084002.

33. Abbott, B.P.; Abbott, R.; Abbott, T.D.; Abernathy, M.R.; Acernese, F.; Ackley, K.; Adams, C.; Adams, T.; Addesso, P.; Adhikari, R.X.; et al. LIGO scientific collaboration and virgo collaboration. GW151226: Observation of gravitational waves from a 22-Solar-Mass binary black hole coalescence. Phys. Rev. Lett. 2016, 116, 241103.

34. Kostelecký, V.A.; Melissinos, A.; Mewes, M. Searching for photon-sector Lorentz violation using gravitational-wave detectors. Phys. Lett. B 2016, 761, 1.

35. Schreck, M. Looking for Lorentz violation with gravitational waves. 2016, arXiv:1603.07452.

36. Bonder, Y. Lorentz violation in the gravity sector: The t puzzle. Phys. Rev. D 2015, 91, 125002.

37. Flowers, N.A.; Goodge, C.; Tasson, J.D. Carleton College, Northfield, MN, USA. Unpublished work, 2016.

38. Shao, C.-G.; Tan, Y.-J.; Tan, W.-H.; Yang, S.-Q.; Luo, J.; Tobar, M.E. Bailey, Q.G.; Long, J.C.; Weisman, E.; $\mathrm{Xu}, \mathrm{R} . ;$ et al. Combined search for Lorentz violation in short-range gravity. Phys. Rev. Lett. 2016, 117, 071102.

39. Shao, C.-G.; Tan, Y.-J.; Tan, W.-H.; Yang, S.-Q.; Luo, J.; Tobar, M.E. Search for Lorentz invariance violation through tests of the gravitational inverse square law at short-ranges. Phys. Rev. D 2015, 91, 102007.

40. Kostelecký, V.A.; Long, J.C. Search for Lorentz violation in short-range gravity. Phys. Rev. D 2015, 91, 092003.

41. Panjwani, H.; Carbone, L.; Speake, C.C. Laboratory searches for preferred frame effects. In CPT and Lorentz Symmetry; Kostelecký, V.A., Ed.; World Scientific: Singapore, 2011; Volume V.

42. Bennett, D.; Skavysh, V.; Long, J.C. Search for Lorentz violation in a short-range gravity experiment. In CPT and Lorentz Symmetry; Kostelecký, V.A., Ed.; World Scientific: Singapore, 2011; Volume V.

43. Battat, J.B.R.; Chandler, J.F.; Stubbs, C.W. Testing for Lorentz violation: Constraints on standardmodel-extension parameters via lunar laser ranging. Phys. Rev. Lett. 2007, 99, 241103.

44. Bourgoin, A.; Hees, A.; Bouquillon, S.; Le Poncin-Lafitte, C.; Francou, G.; Angonin, M.-C. Testing Lorentz symmetry with lunar laser ranging. 2016, arXiv:1607.00294.

45. Le Poncin-Lafitte, C.; Hees, A.; Lambert, S. Lorentz symmetry and very long baseline interferometry. 2016, arXiv:1604.01663.

46. Shao, L. Tests of local Lorentz invariance violation of gravity in the standard model extension with pulsars. Phys. Rev. Lett. 2014, 112, 111103.

47. Shao, L. New pulsar limit on local Lorentz invariance violation of gravity in the standard-model extension. Phys. Rev. D 2014, 90, 122009.

48. Hees, A.; Bailey, Q.G.; Le Poncin-Lafitte, C.; Bourgoin, A.; Rivoldini, A.; Lamine, B.; Meynadier, F.; Guerlin, C.; Wolf, P. Testing Lorentz symmetry with planetary orbital dynamics. Phys. Rev. D 2015, 92, 064049.

49. Bailey, Q.G.; Everett, R.D.; Overduin, J.M. Limits on violations of Lorentz symmetry from gravity probe B. Phys. Rev. D 2013, 88, 102001.

50. Hohensee, M.A.; Mueller, H.; Wiringa, R.B. Equivalence principle and bound kinetic energy. Phys. Rev. Lett. 2013, 111, 151102.

51. Hohensee, H.M.; Chu, S.; Peters, A.; Mueller, H. Equivalence principle and gravitational redshift. Phys. Rev. Lett. 2011, 106, 151102.

52. Chung, K.-Y.; Chiow, S.-W.; Herrmann, S.; Chu, S.; Mueller, H. Atom interferometry tests of local Lorentz invariance in gravity and electrodynamics. Phys. Rev. D 2009, 80, 016002. 
53. Mueller, H.; Chiow, S.-W.; Herrmann, S.; Chu, S.; Chung, K.-Y. Atom interferometry tests of the isotropy of post-Newtonian gravity. Phys. Rev. Lett. 2008, 100, 031101.

54. Dimopoulos, S.; Graham, P.W.; Hogan, J.M.; Kasevich, M.A. Testing general relativity with atom interferometry. Phys. Rev. Lett. 2007, 98, 111102.

55. Scaramuzza, N.; Tasson, J.D. Laser gyroscopes, gravity, and the SME. In CPT and Lorentz Symmetry; Kostelecký, V.A., Ed.; World Scientific: Singapore, 2017; Volume VII.

56. Scaramuzza, N.; Tasson, J.D. Carleton College, Northfield, MN, USA. Unpublished work, 2016.

57. Ortolan, A.; Belfi, J.; Bosi, F.; Di Virgilio, A.; Beverini, N.; Carelli, G.; Maccioni, E.; Santagata, R.; Simonelli, A.; Beghi, A.; et al. The GINGER project and status of the GINGERino prototype at LNGS. J. Phys. Conf. Ser. 2016, 718, 072003.

58. Wagner, T.A.; Schlamminger, S.; Gundlach, J.H.; Adelberger, E.G. Torsion-balance tests of the weak equivalence principle. Class. Quantum Grav. 2012, 29, 184002.

59. Jennings, R.J.; Tasson, J.D.; Yang, S. Matter-sector Lorentz violation in binary pulsars. Phys. Rev. D 2015, 92, 125028.

60. Shao, L.; Stairs, I.H.; Antoniadis, J.; Deller, A.T.; Freire, P.C.C; Hessels, J.W.T.; Janssen, G.H.; Kramer, M.; Kunz, J.; Lämmerzahl, C.; et al. Testing gravity with pulsars in the SKA era. In Proceedings of the Advancing Astrophysics with the Square Kilometre Array (AASKA14), Giardini Naxos, Italy, 9-13 June 2014.

61. Altschul, B; Bailey, Q.G.; Blanchet, L.; Bongs, K.; Bouyer, P.; Cacciapuoti, L.; Capozziello, S.; Gaaloul, N.; Giulini, D.; Hartwig, J.; et al. Quantum tests of the Einstein Equivalence Principle with the STE-QUEST space mission. Adv. Space Res. 2015, 501, 55.

62. Bergé, J.; Touboul, P.; Rodrigues, M. Status of MICROSCOPE, a mission to test the Equivalence Principle in space. J. Phys. Conf. Ser. 2015, 610, 12009.

63. Nobili1, A.M.; Shao, M.; Pegna, R.; Zavattini, G.; Turyshev, S.G.; Lucchesi, D.M.; De Michele1, A.; Doravari, S.; Comandi, G.L.; Saravanan T.R.; et al. "Galileo Galilei" (GG): Space test of the weak equivalence principle to $10^{-17}$ and laboratory demonstrations. Class. Quantum Grav. 2012, 29, 184011.

64. Overduin, J.; Everitt, F.; Worden, P.; Mester, J. STEP and fundamental physics. Class. Quantum Grav. 2012, 29, 184012.

65. Kostelecký, V.A.; Vargas, A. Lorentz and CPT tests in hydrogen, antihydrogen, and related systems. Phys. Rev. D 2015, 92, 056002.

66. Kellerbauer, A.; Amoretti, M.; Belov, A.S.; Bonomi, G.; Boscolo, I.; Brusa, R.S.; Büchner, M.; Byakov, V.M.; Cabaret, L.; Canali, C.; et al. AEgIS Collaboration. Proposed antimatter gravity measurement with an antihydrogen beam. Nucl. Instrum. Methods Phys. Res. B 2008, 266, 351.

67. Amole, C.; Ashkezari, M.D.; Baquero-Ruiz, M.; Bertsche, W.; Butler, E.; Capra, A.; Cesar, C.L.; Charlton, M.; Eriksson, S.; Fajans, J.; et al. Description and first application of a new technique to measure the gravitational mass of antihydrogen. Nat. Commun. 2013, 4, 1785.

68. Indelicato, P.; Chardin, G.; Grandemange, P.; Lunney, D.; Manea, V.; Badertscher, A.; Crivelli, P.; Curioni, A.; Marchionni, A.; Rossi, B.; et al. The Gbar project, or how does antimatter fall? Hyperfine Interact. 2014, 228, 141.

69. Cronin, A.D.; Phillips, T.J.; Fischler, M.; Hahn, A.; Volk, J.T.; Yeh, G.P.; Greaves, R.G.; Howe, S.D.; Jackson, G.P.; Lewis, R.; et al. Letter of intent: Antimatter antigravity experiment (AGE) at Fermilab, 6 February 2009.

70. Dittus, H.; Lämmerzahl, C. Tests of the weak equivalence principle for charged particles in space. Adv. Space Res. 2007, 39, 244.

71. Kirch, K.; Khaw, K.S. Testing antimatter gravity with muonium. Int. J. Mod. Phys. Conf. Ser. 2014, 30, 1460258.

72. Bailey, Q.G.; Tso, R. Light-bending tests of Lorentz invariance. Phys. Rev. D 2011, 84, 085025.

73. Turyshev, S.G.; Shao, M. Laser astrometric test of relativity: Science, technology, and mission design. Int. J. Mod. Phys. D 2007, 16, 2191.

74. Bailey, Q.G. Time-delay and Doppler tests of the Lorentz symmetry of gravity. Phys. Rev. D 2009, 80, 044004.

75. Christophe, B.; Andersen, P.H.; Anderson, J.D.; Asmar, S.; Bério, Ph.; Bertolami, O.; Bingham, R.; Bondu, F.; Bouyer, Ph.; Bremer, S.; et al. Odyssey: A Solar System mission. Exp. Astron. 2009, 23, 529.

76. Appourchaux, T.; Burston, R.; Chen, Y.; Cruise, M.; Dittus, H.; Foulon, B.; Gill, P.; Gizon, L.; Klein, H.; Klioner, S.; et al. Astrodynamical space test of relativity using optical devices I (ASTROD I)—A class-M fundamental physics mission proposal for cosmic vision 2015-2025. Exper. Astron. 2009, 23, 491. 
77. Turyshev, S.G.; Lane, B.; Shao, M.; Girerd, A. A search for new physics with the BEACON mission. Int. J. Mod. Phys. D 2009, 18, 1025.

78. Moore, G.D.; Nelson, A.E. Lower bound on the propagation speed of gravity from gravitational Cherenkov radiation. JHEP 2001, 0109, 023.

79. Catalog of the Highest Energy Cosmic Rays. Available online: http://eas.ysn.ru/catalog (accessed on 1 August 2015).

80. Takeda M.; Hayashida, N.; Honda, K.; Inoue, N.; Kadota, K.; Kakimoto, F.; Kamata, K.; Kawaguchi, S.; Kawasaki, Y.; Kawasumi, N.; et al. Small-scale anisotropy of cosmic rays above $10^{19} \mathrm{eV}$ observed with the Akeno Giant Air Shower Array. Astrophys. J. 1999, 522, 225.

81. Bird, D.J.; Corbato, S.C.; Dai, H.Y.; Elbert, J.W.; Green, K.D.; Huang, M.A.; Kieda, D.B.; Ko, S.; Larsen, C.G.; Loh, E.C.; et al. Detection of a cosmic ray with measured energy well beyond the expected spectral cutoff due to cosmic microwave radiation. Astrophys. J. 1995, 441, 144.

82. Cunningham, G.; Edge, D.M.; England, D.; Evans, A.C.; Hollows, J.D.; Hopper, S.J.; Lapikens, L.; Liversedge, B.; Lloyd-Evans, J.; Ogden, J.D.; et al. Catalogue of Highest Energy Cosmic Rays; Wada, M., Ed.; IPCR Itabashi: Tokyo, Japan, 1980; Volume 1.

83. Abbasi, R.U.; Abu-Zayyad, T.; Allen, M.; Amman, J.F.; Archbold, G.; Belov, K.; Belz, J.W.; Benzvi, S.Y.; Bergman, D.R.; Blake, S.A.; et al. High Resolution Fly'S Eye Collaboration. Search for correlations between HiRes stereo events and active galactic nuclei. Astropart. Phys. 2008, 30, 175.

84. Aab, A.; Abreu, P.; Aglietta, M.; Ahn, E.J.; Samarai, I.A.; Albuquerque, I.F.M.; Allekotte, I.; Allen, J.; Allison, P.; Almela, A.; et al. Pierre Auger Collaboration. Searches for anisotropies in the arrival directions of the highest energy cosmic rays detected by the Pierre Auger Observatory. Astrophys. J. 2015, 804, 15.

85. Winn, M.M.; Ulrichs, J.; Peak, L.S.; McCusker, C.B.A.; Horton, L. The cosmic-ray energy spectrum above $10^{17}$ eV. J. Phys. G 1986, 12, 653.

86. Abbasi, R.U.; Abe, M.; Abu-Zayyad, T.; Allen, M.; Anderson, R.; Azuma, R.; Barcikowski, E.; Belz, J.W.; Bergman, D.R.; Blake, S.A.; et al. Indications of intermediate-scale anisotropy of cosmic rays with energy greater than $57 \mathrm{EeV}$ in the northern sky Measured with the surface detector of the Telescope Array experiment. Astrophys. J. 2014, 790, L21.

87. Linsley, J. Catalogue of Highest Energy Cosmic Rays; Wada, M., Ed.; IPCR Itabashi: Tokyo, Japan, 1980; Volume 1.

88. Pravdin, M.I.; Glushkov, A.V.; Ivanov, A.A.; Knurenko, S.P.; Kolosov, V.A.; Makarov, I.T.; Sabourov, A.V.; Sleptsov, I.Y.; Sleptsova, V.R.; Struchkov, G.G. Estimation of the giant shower energy at the Yakutsk EAS array. In Proceedings of the 29th International Cosmic Ray Conference, Pune, India, 3-10 Aug 2005; p. 243.

89. Díaz, J.S.; Kostelecký, V.A.; Mewes, M. Testing relativity with high-energy astrophysical neutrinos. Phys. Rev. D 2014, 89, 043005.

(C) 2016 by the author; licensee MDPI, Basel, Switzerland. This article is an open access article distributed under the terms and conditions of the Creative Commons Attribution (CC-BY) license (http:/ / creativecommons.org/licenses/by/4.0/). 